
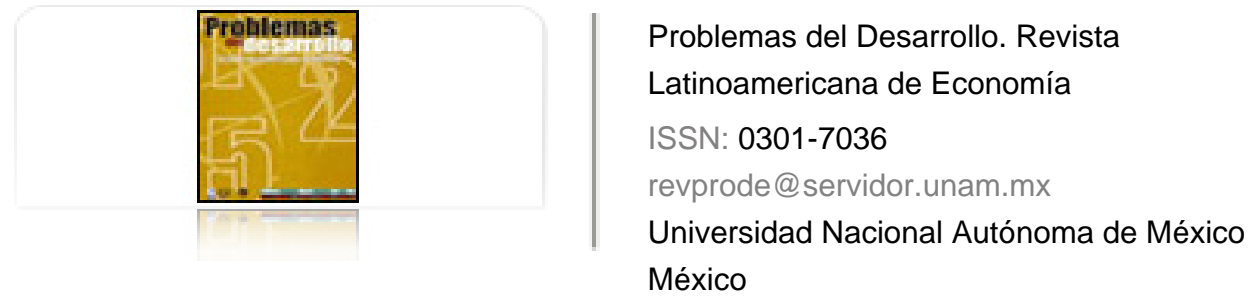

Bañuls Silvera, Víctor Amadeo; Salmerón Silvera, José Luis Áreas clave para desarrollo económico y social: una visión desde la actividad prospectiva internacional

Problemas del Desarrollo. Revista Latinoamericana de Economía, vol. 39, núm. 153, abril-junio, 2008, pp. $139-157$

Universidad Nacional Autónoma de México

Distrito Federal, México

Disponible en: http://www.redalyc.org/articulo.oa?id=11820161006

Cómo citar el artículo

Número completo

- Más información del artículo

Página de la revista en redalyc.org

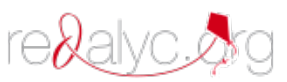

Sistema de Información Científica

Red de Revistas Científicas de América Latina, el Caribe, España y Portugal Proyecto académico sin fines de lucro, desarrollado bajo la iniciativa de acceso abierto 


\title{
ÁREAS CLAVE PARA DESARROLLO ECONÓMICO Y SOCIAL: UNA VISIÓN DESDE LA ACTIVIDAD PROSPECTIVA INTERNACIONAL
}

\author{
Víctor Amadeo Bañuls Silvera* \\ José Luis Salmerón Silvera**
}

Fecha de recepción: 23 de octubre de 2007. Fecha de aceptación: 27 de febrero de 2008.

\section{Resumen}

En el presente trabajo se sitúa la prospectiva tecnológica en el marco del desarrollo económico y social. Ello se fundamenta en la investigación de aspectos clave en el proceso prospectivo como su ámbito y valor estratégico. Una vez establecido el marco de estudio se observa la actividad prospectiva internacional con la técnica denominada análisis de contenido. Concretamente se examinan más de una veintena de actividades prospectivas a lo largo de los cinco continentes. Este estudio tiene como finalidad la plasmación de las prioridades tecnológicas en el ámbito internacional, identificando áreas tecnológicas clave transversales en un contexto global. Dicha aproximación a las áreas clave para el desarrollo económico y social supone una novedad en la literatura.

Palabras clave: prospectiva tecnológica, actividad prospectiva internacional, análisis de contenido, desarrollo económico y social, prioridades tecnológicas, áreas clave para el desarrollo.

* Profesor en Administración y Dirección de Empresas de la Universidad Pablo de Olavide de Sevilla, España. Correo electrónico: vabansil@upo.es.

** Profesor titular en la Universidad Pablo de Olavide de Sevilla, España. Correo electrónico: salmeron@upo.es. 


\section{Summary}

This study situates prospective technology within the framework of economic and social development. This is based on an investigation into the key aspects of the prospective process, such as its environmental and strategic value. Once the framework for the study was established, prospective international activity was seen from the viewpoint of a technique-denominated content analysis. Concretely, more than twenty prospective activities are examined in five continents. The study aims at capturing the technological priorities in the international arena, by identifying key transverse technological areas in a global context. This proximity to the key areas for economic and social development is assumed to be something new in the literature.

Key words: prospective technology, prospective international activity, content analysis, economic and social development, technological priorities.

\section{Résumé}

Dans ce travail, la prospection technologique est placée dans le cadre du développement économique et social. Ceci dans le but de chercher des aspects clés dans le processus prospectiftels que son domaine et sa valeur stratégique. Une fois établi le cadre d'étude, l'activité prospective internationale est observée au moyen de la technique dénommée analyse de contenu. Concrètement, plus d'une vingtaine d'activités prospectives sont examinées à travers les cinq continents. Cette étude a pour fin d'établir les priorités technologiques sur le plan international, en identifiant des domaines technologiques clés transversaux dans un contexte mondial. Cette approche des domaines clés pour le développement économique et social représente une innovation dans la littérature.

Mots clés: prospection technologique, activité prospective internationale, analyse de contenu, développement économique et social, priorités technologiques.

\section{Resumo}

No presente trabalho se situa a prospectiva tecnológica no marco do desenvolvimento econômico e social. Isso se fundamenta na investigação de aspectos chave no processo prospectivo como seu âmbito e valor estratégico. Uma vez estabelecido o marco de estudo se observa a atividade prospectiva internacional com a técnica denominada análise de conteúdo. Concretamente se examinam mais de uma vintena de atividades prospectivas ao longo dos cinco continentes. Este estudo tem como finalidade a plasmação das prioridades tecnológicas no âmbito internacional, identificando áreas tecnológicas chave transversais num contexto global. Tal aproximação às áreas chave para o desenvolvimento econômico e social supõe uma novidade na literatura.

Palavras-chave: prospectiva tecnológica, atividade prospectiva internacional, análise de conteúdo, desenvolvimento econômico e social, prioridades tecnológicas. 


\section{Introducción}

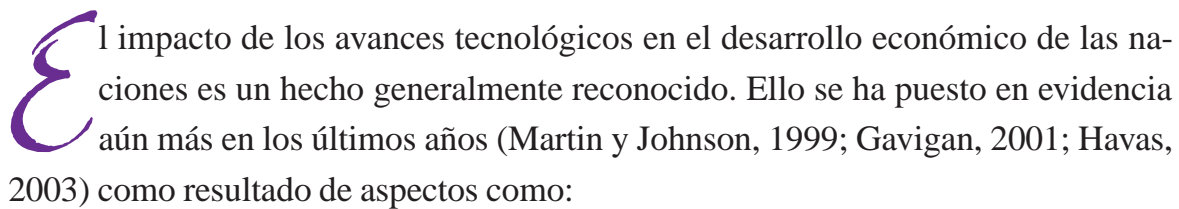

- La intensificación de la competitividad global.

- La internacionalización de la dinámica de la innovación.

- Las nuevas responsabilidades adquiridas por los gobiernos.

- El nacimiento de nuevos tipos de clusters sociales.

- El dinamismo del entorno tecnológico.

- El papel adoptado por los sectores basados en el conocimiento y los servicios.

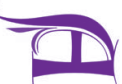

En este nuevo entorno, los actores de los sistemas de innovación nacional tienen que gestionar la incertidumbre para la determinación de las prioridades en inversiones en ciencia, tecnología y desarrollo empresarial. Una de las respuestas de los gobiernos para resolver esta inquietud ha sido la implantación de la prospectiva tecnológica ${ }^{1}$ como herramienta para la ciencia y la tecnología, con la promesa de facilitar la toma de decisiones y con ello dar prioridad a políticas tecnológicas y científicas (Van der Meuler et al., 2003; Keenan, 2003).

La relevancia del ámbito de estudio se pone en evidencia en la multitud de trabajos prospectivos realizados en el escenario internacional en los últimos años (OCDE, 1996, Gavigan y Scapolo, 1999; Rodríguez et al., 2000, Salmerón y Bañuls, 2005). Pese a esta ferviente actividad prospectiva internacional, existe una gran falta de trabajos académicos que aborden aspectos conceptuales, metodológicos y de impacto en las políticas nacionales de desarrollo. Así, los autores pretenden dar una visión genérica del papel de la prospectiva tecnológica en el desarrollo económico y social mediante el análisis de aspectos como su ámbito, valor estratégico y proceso. Además de ello, se estudia la actividad prospectiva internacional por medio de técnicas de análisis de contenido con la finalidad de identificar las áreas clave en el desarrollo económico y social en el mundo. En último término, se profundiza en los resultados derivados de las etapas anteriores, así como tendencias y retos a los que está sujeta esta disciplina.

1 Algunos autores la denominan simplemente prospectiva. En este trabajo, con el ánimo de resaltar su ámbito científico y tecnológico, utilizaremos la acepción prospectiva tecnológica. 


\section{Ámbito de la prospectiva tecnológica}

El origen de la prospectiva tecnológica (FOREN, 2001:5) se sitúa en la convergencia entre la planificación estratégica, la predicción tecnológica y el análisis de políticas (figura 1).

Figura 1

Origen de la prospectiva tecnológica

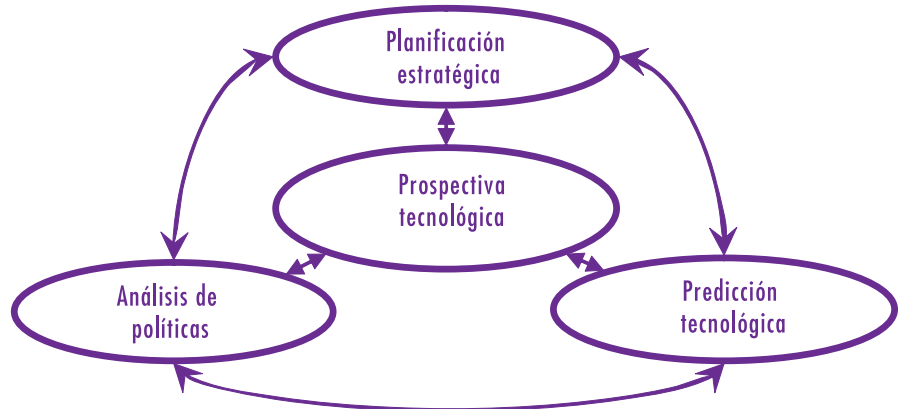

Adaptado de FOREN (2001)

Este origen multidisciplinar se plasma en la definición generalmente aceptada de prospectiva tecnológica, propuesta por Martin (1995:140) que la determina como "el proceso envuelto en el intento sistemático de visualizar el largo plazo de la ciencia y la tecnología, la economía y la sociedad con el ánimo de identificar las áreas estratégicas de investigación así como la aparición de tecnologías genéricas y emergentes que den paso a los mayores beneficios económicos y sociales". Esta definición requiere, a su vez, el establecimiento de los conceptos tecnología genérica y emergente (Martin, 1993).

- Para calificar a una tecnología como genérica se requiere que 1) tenga implicaciones con otro rango de tecnologías y 2) que produzca beneficios en un amplio rango de sectores de la economía o la sociedad.

- Sobre el uso del término emergente, una tecnología emergente es la que, estando suficientemente desarrollada, tiene una alta probabilidad de éxito técnico y comercial en el horizonte temporal.

\section{DeSarrorrollo}


Pese a que la propuesta de Martin ha sido la más difundida, no ha sido la única. A continuación se detallan por orden cronológico algunas de las definiciones más relevantes, incluyendo las más importantes del término prospectiva.

- Ikonicoff, (1973, p. 917): “Se pueden describir tres fases de la prospectiva que corresponden a las tres etapas de toma conciencia progresiva de las sociedades. La primera fase es la de los estudios de coyuntura destinados a facilitar la decisión de inversor [...]. La segunda fase es la de la previsión a medio plazo. En general son previsiones realizadas por los organismos oficiales con el fin de descubrir los desequilibrios o cuellos de botella que puedan producirse en el futuro [...]. El pasaje a la tercera etapa de la prospectiva se produce en el momento en el que el horizonte de aspiraciones de la comunidad se traduce en un proyecto social"'.

- Coates (1985, p. 30): "La prospectiva es el proceso de crear una comprensión y apreciación de la información generada obtenida de mirar hacia el frente. La prospectiva incluye medidas cuantitativas y cualitativas para controlar pistas e indicadores de tendencias y desarrollos envolventes".

- Gavigan (2001, p. 107): "En los últimos años, la prospectiva se ha convertido en un término de uso general para describir una serie de combinaciones de análisis prospectivos y de estudios participativos, destinados a apoyar la toma de decisiones sobre políticas mejor informadas y más eficientemente implementadas. La prospectiva implica de manera fundamental la reunión de agentes claves y de fuentes de conocimiento con el fin de desarrollar puntos de vista estratégicos e inteligencia anticipatoria".

- Rodríguez (2001, p. 15): "La prospectiva tecnológica (1) desarrolla visiones del futuro sobre tecnologías y aspectos clave del desarrollo, (2) proporciona fuentes de conocimiento, (3) posibilita el diálogo entre actores, (4) fomenta la creación de redes de colaboración, (5) proporciona información para el desarrollo de políticas tecnológicas, (6) moviliza un amplio colectivo y le obliga a reflexionar sobre el futuro".

- Van der Meuler et al. (2003, p. 219): "La prospectiva es más que un pronóstico o una predicción. Tiene la misión de gestionar la incertidumbre a través de la continua interacción con los grupos de interés".

- Havas (2003, p. 179): "La prospectiva tecnológica es un proceso sistemático de evaluación de desarrollos científicos y tecnológicos, que puedan impactar en la competitividad industrial, en la creación de salud y en la calidad de vida". 
- Salo y Cuhls (2003, p.79): "La prospectiva tecnológica es un instrumento de la inteligencia política estratégica que busca generar un conocimiento acerca de los posibles desarrollos científicos y tecnológicos y sus impactos sobre la economía y la sociedad para dar soporte a la determinación de las políticas científicas y tecnológicas, el alineamiento de la investigación y los desarrollos con las necesidades sociales, la intensificación de las colaboraciones de investigación y desarrollos y los sistemáticos desarrollos en el largo plazo de sistemas de innovación".

- Lattre Gasquet et al. (2003, p.217): "La prospectiva es una poderosa herramienta para imaginar futuros potenciales, aumentar la conciencia pública, ayudar a la toma de decisiones y afrontar cuestiones referentes a relaciones entre ciencia y tecnología”.

De las aproximaciones al concepto de prospectiva tecnológica citadas, se pueden extraer los siguientes componentes para el establecimiento de su ámbito (cuadro 1):

- La prospectiva tecnológica es un proceso sistemático y continuado, no es un acto puntual y aislado.

- Es un proceso participativo, fruto del diálogo entre los actores de los sistemas de innovación nacionales, tales como tecnólogos, científicos, decisores públicos y agentes sociales.

- El alcance de la prospectiva tecnológica es muy amplio, comprendiendo no sólo tecnologías y sus aplicaciones, sino el establecimiento de políticas públicas y los retos sociales que éstas conllevan.

- La finalidad que se persigue radica en el fomento del pensamiento a largo plazo de los grupos de interés, la identificación de oportunidades de acciones conjuntas y el ofrecimiento de recomendaciones para el desarrollo económico y social.

Cuadro 1

Ámbito de la prospectiva tecnológica

\begin{tabular}{ll}
\hline Alcance & Ciencia, tecnología, economía y sociedad \\
Campos relacionados & $\begin{array}{l}\text { Predicción tecnológica, planificación estratégica, análisis de políticas, } \\
\text { gestión de la tecnología, gestión de la innovación }\end{array}$ \\
Periodicidad & Proceso sistemático y continuado \\
Usuarios & Actores de los sistemas de innovación, grupos de interés \\
Finalidad & Identificación de tecnologías emergentes, oportunidades de acciones \\
& coniuntas y recomendaciones para el desarrollo económico y social
\end{tabular}


Una vez establecido el ámbito de la prospectiva tecnológica, lo vamos a delimitar con el análisis de sus particularidades frente al enfoque clásico de anticipación al futuro: la predicción tecnológica.

\section{Predicción y prospectiva tecnológica}

La predicción y prospectiva tecnológicas están en la práctica íntimamente ligadas. Ello se debe a que la prospectiva tecnológica se fundamenta en la identificación de las diferentes opciones para el futuro, basándose en técnicas de predicción tecnológica. No obstante, existen diferencias apreciables entre ambas.

Martino (1983) define la predicción tecnológica como "una predicción acerca de las características futuras de maquinaria, procedimientos o técnicas". De esta definición clásica se deriva el primer hecho diferenciador entre predicción y prospectiva

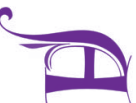
tecnológica: la primera termina en la identificación de la probabilidad asociada a distintos futuros, mientras que la segunda no se limita a predecir cual será el futuro tecnológico esperado. La prospectiva tecnológica describe una variedad de futuros potenciales, para contribuir a conseguir unos resultados concretos por parte de los grupos de interés (Cuhls, 2003).

El segundo hecho diferenciador entre ambos conceptos es que mientras la mayoría de los enfoques tradicionales de predicción tecnológica se centran en la precisión de predicciones a largo plazo, la prospectiva tecnológica se centra en estimular la implicación activa de los participantes. Este énfasis en el proceso, más que en el resultado, ha sido justificado (Martin e Irving, 1989) por los beneficios que dicho proceso prospectivo conlleva, mediante las cinco $C$ :

- Comunicación: reuniendo los grupos dispares en un nuevo foro en que ellos pueden actuar de manera recíproca.

- Concentración: alentando a los participantes implicados en el proceso a pensar en términos de largo plazo.

- Coordinación: para que las personas diferentes puedan formar las sociedades de investigación y desarrollo (I+D) productivas.

- Consenso: creando una visión compartida del futuro que a los participantes les gustaría lograr.

- Compromiso: asegurando que los individuos participan totalmente y puedan llevar a cabo los cambios en la dirección del ejercicio de prospectiva tecnológica. 
Estas cinco $C$ también son conocidas como "resultados del proceso", en contraposición al término "resultados tangibles", empleado para las conclusiones del proceso de prospectiva, plasmadas en informes, conferencias o grupos de trabajo. Esta denominación se debe a que algunos autores argumentan que, más allá de los beneficios del proceso prospectivo, las cinco $C$ son resultados del proceso prospectivo en sí mismo.

Esta focalización en el proceso, más que en los resultados, ha derivado en que, en comparación con los estudios de predicción tecnológica, las investigaciones de prospectiva tecnológica han puesto poco énfasis sobre la clase de futuro que está construyendo y su validez. Con frecuencia, estas cuestiones quedan relegadas tras una argumentación que indica que la intención de la prospectiva no es una predicción del futuro y que el proceso de interacción es tan relevante como el resultado. Sin embargo, estudios recientes reivindican la orientación de la investigación de la prospectiva a su integración con instrumentos metodológicos provenientes de campos anexos para incrementar su precisión (Bañuls y Salmerón, 2007a; Bañuls y Salmerón, 2007b, Bañuls y Salmerón, 2008).

A continuación se exponen los motivos para realizar prospectiva tecnológica en lugar de predicción tecnológica mediante el análisis del valor estratégico de la primera.

\section{Valor estratégico de la prospectiva tecnológica}

En comparación con el enfoque de predicción tecnológica, la literatura de prospectiva tecnológica ha puesto poca atención sobre su actual valor estratégico. En sus orígenes, el impacto de la prospectiva tecnológica fue medido en términos de número de actividades y actores involucrados en el proceso.

Esta falta de interés se materializa en la escasez de estudios acerca del valor estratégico de la prospectiva tecnológica (Major et al., 2001; Van der Meulen et al., 2003). No obstante, existen algunos trabajos enfocados a justificar dicho valor estratégico.

Una primera aproximación al valor estratégico de la prospectiva fue realizada por Godet (1994; 2000), a partir de los enfoques tradicionales de planificación estratégica (Ansoff, 1965; Ackoff, 1971). Dicho autor encuentra el valor estratégico de la prospectiva en 1) servir de herramienta de anticipación del futuro a la planificación estratégica, 2) al gestionar la incertidumbre del proceso de toma de decisiones estratégicas en el horizonte temporal y 3) al promover la acción conjunta de los actores implicados en dicha estrategia. Al seguir esta aproximación fundamentada en la literatura de estrategia organizativa, otros autores han justificado el valor de la prospectiva en 
el sostenimiento de la ventaja competitiva de las organizaciones (Anderson, 1997) o como competencia básica de las mismas (Major et al., 2001).

Otra corriente de pensamiento en la que se ha fundamentado el valor estratégico de la prospectiva ha sido la dinámica de las expectativas (De Laat, 1996). Este enfoque muestra cómo los actores usan expectativas o creencias acerca de sus posiciones futuras vis à vis con el resto de actores y competidores, creando condiciones de soporte para las actividades de innovación. El resultado es un mutuo posicionamiento de los actores y el desarrollo de estrategias innovadoras soportadas por múltiples actores (Van der Meulen, 1999).

Por último, está la perspectiva de evaluación de los resultados del proceso de prospectiva tecnológica mediante la medición del impacto de los mismos. En este ámbito, destaca el trabajo de Keenan (2000), quien realiza un estudio evaluativo en la instauración del proyecto de prospectiva tecnológica en Reino Unido. En este trabajo se traza el origen de un número de prioridades que arrojó el estudio de prospectiva tecnológica en dicho país, en donde describe el modo en que diferentes actores (universidad, industria y gobierno) se organizaban para la implantación de esas prioridades (Keenan, 2000). Esta evaluación resalta en el valor añadido de la prospectiva tecnológica de los "resultados del proceso" de la misma, principalmente en la creación de redes y el efecto sinérgico de dicho proceso.

\section{Taxonomía de procesos de prospectiva tecnológica}

La taxonomía de procesos de prospectiva se puede analizar en función de tres categorías (Martin, 1995): la organización que los realiza, el grado de especificidad y el horizonte temporal (cuadro 2).

Con respecto a la primera categoría, el proceso de prospectiva tecnológica puede ser realizado por:

- Instituciones públicas, bien de manera centralizada por el gobierno o coordinada desde un organismo público independiente.

- Centros de investigación, tanto públicos como privados, ubicados en universidades, parques tecnológicos y otras instituciones científicas.

- Asociaciones industriales, empresas con base tecnológica y otros centros de ámbito privado.

Un segundo criterio sería el grado de especificidad. En función de esta categoría pueden ser: 
Cuadro 2

Taxonomía de prospectiva tecnológica

\begin{tabular}{ll}
\hline Categoría & Características \\
\hline Organización que los realiza & Gobierno \\
& Organismos públicos independientes \\
& Centros de investigación \\
& Asociaciones industriales \\
& Empresas con base tecnológica \\
Grado de especificidad & Holística \\
& Macronivel \\
& Mesonivel \\
& Micronivel \\
& Corto plazo \\
& Medio plazo \\
& Largo plazo \\
\hline
\end{tabular}

- Holísticos, es decir, que abarcan todo el espectro de la ciencia y la tecnología.

- Macronivel, cuando se cubre un determinado número de áreas tecnológicas.

- Mesonivel, cuando se concentra en una determinada área científica o tecnología.

- Micronivel, cuando el ámbito se circunscribe en una tecnología concreta, un proyecto de investigación o incluso un producto.

Los procesos de prospectiva tecnológica se pueden segmentar también en cuanto a su horizonte temporal. Éste puede estar situado en el corto, el medio o el largo plazos. No obstante, resulta más habitual el largo plazo.

\section{La actividad de prospectiva tecnológica}

Pese a la relativamente reciente eclosión de la actividad prospectiva en el ámbito internacional, sus orígenes se remontan a la década de sesenta en EU. Por una parte, en las actividades realizadas por el Comité de Ciencia y Política Pública (COSPUP²), orientadas a la identificación de las áreas de investigación más prometedoras (Martin, 1995). Por otra, en los desarrollos metodológicos de la corporación RAND, especialmente del método Delphi (Grupp y Linstone, 1999).

2 Acrónimo anglosajón de Committee on Science and Public Policy.

\section{Desarrollo}


No obstante, estas actividades pioneras de prospectiva del gobierno estadounidense no llegaron a consolidarse y desaparecieron casi por completo en los años setenta. Es precisamente a mediados de esta década cuando en el seno del Instituto Japonés de Ciencia y Tecnología (NISTEP³) se inició una de las actividades prospectivas más representativas en el ámbito internacional. Dicha actividad, la cual se continúa en nuestros días con una periodicidad de cinco años, consiste en un estudio prospectivo acerca del futuro de la ciencia y tecnología de Japón. Otras iniciativas pioneras son las realizadas en Alemania y Francia (Irving y Martin, 1984).

Sin embargo, en la década de los noventa se produce un incremento notable de las actividades de prospectiva tecnológica, tanto en el ámbito nacional como supranacional. Las actividades prospectivas de los últimos 15 años se diferencian de sus antecedentes de los setenta y ochenta porque resaltan la exploración sistemática de las dinámicas futuras y la importancia de las interacciones entre actores de distintas procedencias en los sistemas de innovación nacional. En lugar de reducir la incertidumbre mediante la planificación, la prospectiva tecnológica promete la gestión de este problema mediante una intensiva interacción con los grupos de interés.

Europa Occidental y el conglomerado de países Asia-Pacífico han sido las áreas donde mayor difusión ha tenido la prospectiva tecnológica. Dicha actividad fue fuertemente influenciada en ambos casos por el Delphi Japonés; en el caso de Europa Occidental, por transferencia de experiencias niponas en el contexto alemán; en el caso de los países de Asia-Pacífico, por la propia influencia cultural japonesa en el modelo de desarrollo de los países de la zona. Sin embargo, el paso del tiempo ha propiciado el surgimiento de enfoques prospectivos distintos al japonés, siendo hoy día la única la identidad de cada proceso de prospectiva tecnológica. Además, ahora no se puede acotar esta tarea en un área mundial concreta, pues está localizada en los cinco continentes. De la misma manera, no se puede asociar en exclusiva la prospectiva tecnológica a los países desarrollados, ya que también se utiliza en naciones en vías de desarrollo.

Otro indicador relevante de la prospectiva tecnológica en las áreas en la actividad científico-tecnológica en el escenario internacional es la creación de instituciones supranacionales cuya última finalidad es coordinar la actividad prospectiva en sus áreas de influencia. Éste es el caso del Instituto de Estudios de Prospectiva Tecnológica $\left(\mathrm{IPTS}^{4}\right)$ en la Unión Europea y del Centro de Prospectiva Tecnológica del área

Acrónimo anglosajón de National Institute of Science and Technology Policy.

Acrónimo anglosajón de Institute for Prospective Technological Studies. 
de Cooperación Económica de Asia-Pacifico $\left(\mathrm{APEC}^{5}\right)$. Además, cabe destacar a este respecto que otras organizaciones supranacionales ya existentes, como la Organización para la Cooperación y el Desarrollo Económicos (OCDE) y la Organización de Naciones Unidas (ONU), han llevado a cabo de modo regular proyectos de prospectiva tecnológica.

En países hispanoparlantes, al igual que en el resto del mundo, ha proliferado la actividad prospectiva. En América Latina y Caribe, ésta ha sido promovida principalmente por la ONUDI ${ }^{6}$ y el nodo latinoamericano del Millenium Project de la ONU. Sin embargo, existen otras actividades cercanas a la prospectiva paralelas a los desarrollos realizados por estas entidades supranacionales en América Latina y Caribe, como los de Argentina, Bolivia, Chile, Colombia, Costa Rica, Uruguay y Venezuela (Rodríguez et al., 2000). En España, el centro de la actividad prospectiva es el Observatorio de Prospectiva Tecnológica Industrial (OPTI) (OPTI 1999, 2000, 2001). Otros estudios que han abordado tendencias tecnológicas futuras y su impacto en la sociedad española con un enfoque prospectivo son los informes anuales de la Fundación Cotec (i.e. 2002, 2003), y de la fundación Telefónica (i.e. 2002, 2003), entre otros (Héraud y Cuhls, 1999; Mañá, 2001).

\section{Áreas clave para el desarrollo económico y social}

En este epígrafe se analizan las áreas clave para el desarrollo económico de las naciones, según su relevancia en la actividad prospectiva internacional. Para ello se estudiará una muestra de actividades prospectivas en el mundo.

La muestra de procesos prospectivos tecnológicos se ha tomada de la base de datos del proyecto alemán Futur. Éste es una iniciativa del ministerio alemán de educación e investigación, que cuenta con un resumen de las actividades prospectivas más relevantes en el ámbito internacional. Para analizar dicha muestra se ha utilizado la técnica denominada análisis de contenido. En seguida se estudian los detalles en la aplicación de dicha técnica.

\section{Metodología de análisis}

El análisis de contenido es una técnica de investigación destinada a formular, a partir de ciertos datos, inferencias reproducibles y válidas que puedan aplicarse a su contexto

Acrónimo anglosajón de Asia-Pacific Economic Cooperation.

Acrónimo de Organización de Naciones Unidas para el Desarrollo Industrial.

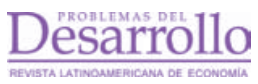


(Krippendorff, 1990 p.28). Esta metodología se ha seleccionado debido a su adecuación para procesar datos científicos. A continuación se detallan algunos aspectos concernientes a esta metodología como las unidades de muestreo y registro y la codificación.

Las unidades de muestreo, definidas como las porciones de la realidad observada que se consideran independientes unas de otras, son las actividades de prospectiva tecnológica internacionales con un grado de especificidad de macronivel. Nótese que se excluyen 1) las actividades realizadas por organismos supranacionales, por evitar solapamientos entre actividades prospectivas; 2) las actividades holísticas por no encontrarse dividas en áreas clave; y 3) las mesonivel y micronivel por centrarse en un área concreta. En caso de existir dos o más actividades internacionales en un país con dichas características, se ha seleccionado la más reciente. El resultado final son las 27 unidades de muestreo recogidas en el cuadro 3.

Cuadro 3

Muestra de actividades prospectivas internacionales

\begin{tabular}{ll}
\hline País & Programa \\
\hline Alemania & Second comprehensive study on the development of science and technology \\
Australia & Matching science and technology to future needs 2010 \\
Austria & Technology Delphi \\
Bélgica & Belgian Federal Foresight Study \\
Corea del sur & Second Technology Forecast \\
China & Technology Foresight 2003 \\
EU & New Forces at Work: Industries Views Critical Technologies \\
Eslovaquia & Technology Foresight Slovakia 2015 \\
España & Informes de Prospectiva Tecnológica Industrial \\
Estonia, Malta, Chipre & e-FORESEE \\
Filipinas & Science and Technology Master Plan (STMP) \\
Finlandia & On the way to technology vision \\
Francia & List of Key Technologies (Technologies Clés) \\
Grecia & The Greek Technology Foresight Programme (TF) \\
Holanda & Dinamo 2003-2006 \\
Hungría & Technology Foresight Programme (TEP) \\
India & Technology Vision 2020 \\
Irlanda & Technology Foresight Ireland \\
Israel & Science and Technology Foresight for Israel \\
Japón & Technology Foresight. Future technology in Japan \\
Nueva Zelanda & The Foresight Project \\
Portugal & Engenharia e Tecnologia 2000 (ET 2000) \\
Reino Unido & The Foresight Programme \\
República Checa & Analyses of international key technologies lists \\
Sudáfrica & National Research and Technology Foresight Project (NRTF) \\
Suecia & 2 Teknisk Framsyn \\
Turquía & Foresight Turkey - Vision 2023 \\
\hline
\end{tabular}


Las unidades de registro, definidas como fracciones de las unidades anteriores que determinan la codificación de las diferentes categorías, son cada una de las áreas clave halladas como explicación en cada los procesos de prospectiva. En total se identificaron 288 unidades de registro entre las 27 unidades de muestreo.

La codificación se ha realizado con base en la lista de áreas clave del octavo proceso de prospectiva tecnológica japonés (cuadro 4). Dicho proceso ha sido seleccionado como base de la codificación por ser el más reciente de la actividad prospectiva nacional con grado de especificidad macronivel más influyente en el ámbito internacional (Grupp y Linstone, 1999).

Cuadro 4

Categorización de áreas clave

\begin{tabular}{ll}
\hline Área clave & Ejemplos de grupos especificos de interés \\
\hline Información y comunicaciones & Interfaz humano-máquina \\
& Seguridad de la información \\
& Nuevos principios para la información y las comunicaciones \\
& Computación ubicua \\
& Tecnología software \\
& Electrónica del silicio \\
& Dispositivos ópticos y fotoelectrónicos \\
& Electrónica sin hilos \\
Electrónica & Electrónica molecular y orgánica \\
& Robótica \\
Ciencias de la vida & Generación y crecimiento cerebral \\
& Funciones cerebrales de alto nivel \\
& Control de funciones biológicas de alto nivel \\
& Biología ambiental y ecológica \\
& Nanobiología \\
& Medicina personalizada \\
Salud, medicina y bienestar & Mecanismos de defensa biológica y aplicación terapéutica \\
& Medicina preventiva \\
Medidas contra la enfermedades infecciosas que reaparecen & Medicina y bienestar para la sociedad anciana \\
Interacción entre biodiversidad y ecosistema \\
Soluciones biológicas al problema ambiental \\
Sociedad sostenible \\
Descarrollo de la tecnología de la producción armonizada con el ecosistema \\
Sistema alimentarios seguros y sostenibles \\
\hline
\end{tabular}

Continúa 


\begin{tabular}{|c|c|}
\hline Área clave & Ejemplos de grupos especificos de interés \\
\hline \multirow[t]{5}{*}{ Fronteras } & Tecnología de exploración planetaria \\
\hline & Tecnología básica para transporte espacial \\
\hline & Tecnología para la observación exacta del entorno terráqueo \\
\hline & Profundidades terrestres \\
\hline & Océano y fondo marino \\
\hline \multirow[t]{5}{*}{ Recursos y energía } & Energía nuclear innovadora \\
\hline & Sistemas de energía del hidrógeno \\
\hline & Células de combustible \\
\hline & Sistemas de energía descentralizados \\
\hline & Energías renovables \\
\hline \multirow[t]{5}{*}{ Entorno } & Entorno global \\
\hline & Entorno urbano \\
\hline & Îndices económicos ambientales \\
\hline & Desastres ambientales \\
\hline & Recursos de agua \\
\hline \multirow[t]{5}{*}{ Nanotecnología y materiales } & Simulación de modelado de nanomateriales \\
\hline & Medidas y tecnología de análisis \\
\hline & Tecnología de síntesis y de proceso \\
\hline & Nuevos materiales para el control de la estructura a nanonivel \\
\hline & Nanodispositivos y sensores \\
\hline \multirow[t]{5}{*}{ Manufactura } & Diseño virtual \\
\hline & Tecnología de la fabricación para los productos de alto valor añadido \\
\hline & Tecnología de la fabricación en ambientes especiales \\
\hline & Tecnología de reciclado \\
\hline & Tecnología superficial de la modificación y del control de la interfaz \\
\hline \multirow[t]{5}{*}{ Infraestructura industrial } & Optimización de industrial \\
\hline & Dispersión y concentración industrial \\
\hline & Gestión del conocimiento \\
\hline & Toma de decisiones corporativas \\
\hline & Gestión del riesgo \\
\hline \multirow[t]{5}{*}{ Infraestructura social } & Revitalización, mantenimiento y gestión de la infraestructura social \\
\hline & Tecnología ambiental y arquitectura \\
\hline & Sistemas del transporte \\
\hline & Tráfico \\
\hline & Sistemas de la logística \\
\hline \multirow[t]{5}{*}{ Tecnología Social } & Seguridad y estabilidad de la vida cotidiana \\
\hline & Seguridad urbana \\
\hline & Tecnologías de la educación \\
\hline & Tecnologías de preservación cultural \\
\hline & Evaluación de la tecnología \\
\hline
\end{tabular}

Adaptado de NISTEP, 2066. 
La labor de codificación fue realizada por los autores. Estos codificadores cumplen los requisitos de nivel cultural, formación y conocimiento del marco teórico. El coeficiente de fiabilidad de la codificación (CF) (expresión 1) en la primera ronda ha sido de $75.82 \%$. Al seguir la metodología de análisis de contenido, en la segunda ronda se revisaron las divergencias. Tras dicha revisión el grado de acuerdo entre codificadores fue de $100 \%$.

donde:

$$
\mathrm{CF}_{1}=2 \mathrm{~m} /\left(\mathrm{N}_{1}+\mathrm{N}_{2}\right)
$$

"m" es el número de decisiones de codificación en las que coinciden el codificador 1 y el codificador 2 .

" $\mathrm{N}_{1}$ " es el número de decisiones de codificación realizadas por el codificador 1.

" $\mathrm{N}_{2}$ " es el número de decisiones de codificación realizadas por el codificador 2.

A continuación se analizan los resultados de la aplicación del análisis de contenido.

\section{Resultados}

En el cuadro 5 se muestran los resultados por unidad de muestreo.

\begin{tabular}{lcc} 
& $\begin{array}{c}\text { Cuadro 5 } \\
\text { Resultados }\end{array}$ & \\
\hline Área Clave & & \\
\hline Información y comunicaciones & Procesos & $\%$ \\
Nanotecnología y materiales & 24 & $88.89 \%$ \\
Recursos y energía & 23 & $85.19 \%$ \\
Infraestructura social & 21 & $77.78 \%$ \\
Manufactura & 20 & $74.07 \%$ \\
Agricultura, forestación, pesca y alimentación & 20 & $74.07 \%$ \\
Infraestructura industrial & 19 & $70.37 \%$ \\
Salud, medicina y bienestar & 19 & $70.37 \%$ \\
Entorno & 19 & $70.37 \%$ \\
Tecnología social & 17 & $62.96 \%$ \\
Ciencias vivas & 17 & $62.96 \%$ \\
Fronteras & 13 & $48.15 \%$ \\
Electrónica & 10 & $37.04 \%$ \\
\end{tabular}

Con este análisis se puede apreciar cómo "Información y comunicaciones" es el área clave que en mayor número de procesos de prospectiva tecnológica se encuentra

\section{Desarrollo}


presente $(88.89 \%)$. Esta clasificación viene seguida de "Nanotecnología y materiales" (85.19\%), así como de "Recursos y energía" (77.78\%). Nótese que todas las áreas clave tienen una presencia igual o superior a $50 \%$ en la muestra, excepto "Ciencias de la vida" (48.15\%), "Fronteras" (37.04\%) y "Electrónica" (25.93\%).

\section{Conclusiones}

En este artículo se realiza una aproximación a las áreas clave para el desarrollo económico y social desde el análisis de la actividad internacional en materia de prospectiva tecnológica. Es la primera vez que se realiza un análisis de esta magnitud con técnicas para el tratamiento de contenidos, por lo que la presente investigación supone una contribución al campo de la prospectiva tecnológica.

Como resultado del análisis se deduce que la "Información y las comunicacio-

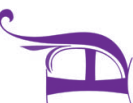
nes", "Nanotecnología y materiales", "Recursos y energía" y "Salud, medicina y bienestar", entre otras (cuadro 5), son consideradas como áreas estratégicas para el desarrollo económico internacional. Estos resultados han de ser interpretados en sentido amplio, pues cada país prioriza su esfuerzo en investigación e inversión en función de su posición competitiva, así como de su papel en la economía internacional. No obstante, se puede interpretar que existen áreas clave transversales a cualquier economía en un contexto global.

Una segunda contribución del artículo es el análisis de la actividad en prospectiva tecnológica internacional, contextualizado con aspectos como el ámbito, el valor estratégico y el proceso de la prospectiva tecnológica. Fruto del estudio de dicha actividad se desprende la relevancia de la actividad prospectiva en países asiáticos y europeos. Esta relevancia se debe al fomento que organizaciones gubernamentales y supranacionales han realizado de la actividad prospectiva en las dos últimas décadas. En el resto del mundo, está actividad ha sido más esporádica.

Al centrarnos en el caso de América Latina, la actividad prospectiva ligada a órganos de poder ha sido promovida por divisiones específicas de organizaciones supranacionales. Al extrapolar otras acciones de organismos supranacionales como IPTS en Europa o APEC en Asia-Pacífico, se prevé que en los próximos años dicha actividad redunde en la asimilación de la prospectiva tecnológica por parte de los gobiernos latinoamericanos como una herramienta estable de inteligencia política. 


\section{Bibliografía}

Ackoff, Rusell, A Concept of Corporate Planning, Nueva York, John Wiley \& Sons Inc, 1971.

Anderson, Joe, "Technology Foresight for Competitive Advantage", Long Range Planning, vol. 30, octubre de 1997, pp. 665-677.

Ansoff, Igor, Corporate Strategy, Nueva York, McGraw-Hill, 1965.

Bañuls, Víctor Amadeo y Salmerón, José Luís, "Benchmarking the Information Society in the Long Range", Futures, vol. 39, núm. 1, 2007a, pp. 83-95.

, "A Scenario-based Assessment Model-SBAM", Technological Forecasting and Social Change, vol. 74, núm. 6, 2007b, pp. 750-762.

"Foresighting key areas in the information technology industry", Technovation vol. 28, núm. 3, 2008, pp. 103-111.

Coates, Joseph. "Foresight in Federal Government Policymaking", Futures Research Quarterly, verano de 1985, pp. 29-53.

Culhs, Kerstin, "Twelve From Forecasting to Foresight Processes-New Participative Foresight Activities in Germany", Journal of Forecasting, vol. 22, núm. 2-3, 2003, pp. 93-111.

De Laat, Bastiaan. Scripts for the Future. Technology Foresight, Strategic Evaluation and Socio-technical Networks: the confrontation of script-based scenarios, tesis doctoral, Amsterdam, University of Amsterdam, 1996.

FOREN, A practical Guide to Regional Foresight. European Commision Research Directorate General, Informe EUR 20128 EN, European Communities, 2001.

Fundación COTEC, Tendencias Tecnológicas en Europa: Análisis de los Procesos de Prospectiva, Madrid, Fundación COTEC, 2003.

Fundación Telefónica, La Sociedad de la Información en España 2002: Presente y Perspectivas, Madrid, Fundación Telefónica, 2003.

Gavigan, James, "Panorama de la Prospectiva en Europa. Principios y Visión General por Países", Economía Industrial, vol. 342, núm. 6, 2001, pp. 107-118.

y Scapolo, Fabiana, "Matching Methods to the Mission: a Comparison of $\mathrm{Na}-$ tional Foresight Exercises", Foresight, vol. 1, núm. 6, 1999, pp. 495-517.
Godet, Michel, From Anticipation to Action, París, UNESCO Publishing, 1994.

, La Caja de Herramientas de la Prospectiva Estratégica. Cuadernos del LIPS, $4^{\mathrm{a}}$ ed., París, Gerpa, 2000.

Grupp, Hariolf y Linstone, Harold, "National Technology Foresight Activities around the Globe: Resurrection and New Paradigms", Technological Forecasting and Social Change, 60, 1999, pp. 85-94.

Havas, Attila, "Evolving Foresight in a Small Transition Economy", Journal of Forecasting, vol. 22, núm. 2/3, 2003, pp. 179-201.

Héraud, Jean-Alain y Cuhls, Kerstin, "Current Foresight Activities in France, Spain, and Italy", Technological Forecasting and Social Change, 60, 1999, pp. 55-70.

Ikonicoff, Moisés, "Las Etapas de la Prospectiva”, Desarrollo Económico, vol. 12, núm. 48, 1973, pp. 915-921.

Irving, John y Martin, Ben, Foresight in Science: Picking the Winners. London, Pinter, 1984.

Keenan, Michael, An Evaluation of the Implementation of the UK Technology Foresight Programme, tesis doctoral, University of Manchester, 2000.

"Identifying Emerging Generic Technologies at the National Level: the UK Experience", Journal of Forecasting, vol. 22, núm. 2/3, 2003, pp. 129-160.

Krippendorf, Klaus, Metodología de Análisis de Contenido: Teoría y Práctica, Barcelona, Ediciones Paidós, 1990.

Lattre-Gasquet, Marie, Petithuguenin, Philippe y Sainte-Beuve, Jerome, "Foresight in a Research Institution: a Critical Review of Two Exercises", Journal of Forecasting, vol. 22, núm. 2-3, 2003, pp. 203-210.

Major, E., Asch, D. y Cordey-Hayes, M., "Foresight as a core competence", Futures, vol. 33, 2001, pp. 91-107.

Mañá, Francesc, "Tendencias Tecnológicas en la Sociedad de la Información", Economía Industrial, vol. 342, núm. 6, 2001, pp. 95-105.

Martin Ben, Research Foresight and the Exploitation of the Science Base, Londres, HMSO, 1993.

e Irvine John, Research Foresight, London, Frances Pinter, 1989.

y Johnston Ron, "Technology Foresight for Wiring up the National Innovation System. Experiences in Britain, Australia, 
and New Zealand", Technological Forecasting and Social Change, vol. 60, 1999, pp. 37-54.

"Foresight in Science and Technology", Technology Analysis and Strategic Management, vol. 7, núm. 2, 1995, pp. 139-168.

Martino, Joseph, Technological Forecasting for Decision Making, $2^{\mathrm{a}}$ Ed, New York, Amsterdam, Oxford, North-Holland, 1983.

NISTEP, The Seventh National Japanese Science and Technology Foresight-Future Technology in Japan, Tokio, NISTEP, Informe 71, 2001.

, The Eighth National Japanese Science and Technology Foresight-Future Technology in Japan, NISTEP, Informe 97, Tokio, 2006.

OCDE, "Special Issue on Government Technology Foresight Exercises", STI Review, 17, 1996.

OPTI, Primer Informe de Prospectiva Tecnológica Industrial, Madrid, OPTI, 1999.

, Segundo Informe de Prospectiva Tecnológica Industrial, Madrid, OPTI, 2000.

, Tercer Informe de Prospectiva Tecnológica Industrial, Madrid, OPTI, 2001.
Rodríguez, Jesús, "Introducción a la Prospectiva: Metodologías, Fases y Explotación de Resultados", Economía Industrial, vol. 342, núm. 6, 2001, pp. 13-20.

Rodríguez, Sandra, Torrealba, Mariana, Cárdenas, Antonio y Dávila, Gulfredo, Inteligencia Tecnológica, Caracas, Los Teques, 2000.

Salo, Ahti y Cuhls, Kerstin, "Technology Foresight-Past and Future", Journal of Forecasting, vol. 22, núm. 2/3, 2003, pp. 79-82.

Salmerón, José Luís y Bañuls, Víctor Amadeo, "Detecting IS/IT Future Trends: An Analysis of Technology Foresight Processes Around the World", Lecture Series on Computer and Computational Sciences, vol. 2, 2005, pp. 120-123.

Van der Meulen, Barend, De Wilt, Jan y Rutten, Hans, "Developing Futures for Agriculture in the Netherlands: a Systematic Exploration of the Strategic Value of Foresight", Journal of Forecasting, vol. 22, núm. 2/3, 2003, pp. 219-233.

, "The Impact of Foresight on Environmental Science and Technology Policy in the Netherlands", Futures, vol. 31, 1999 , pp. 7-23. 